Laboratorio de Arte, 7-1994 http://dx.doi.org/10.12795/LA.1994.i07.17

\title{
EL ARTESONADO DEL REFECTORIO DE LA CARTUJA DE SANTA MARÍA DE LAS CUEVAS DE SEVILLA
}

\author{
POR Maa MERCEDES FERNÁNDEZ MARTín
}

En los últimos años se han intensificado considerablemente los estudios sobre la carpintería de armar o de lo blanco, de la que tan abundantes ejemplos existen en todo el territorio español. Entre esos trabajos sobresalen los llevados a cabo por Nuere Mateuco quien, además de facilitar considerablemente el estudio y tipología de estas armaduras, ha realizado un inventario de las mismas, dando a conocer gran número de piezas '. Así Andalucía, junto a la comunidad de Castilla y León, es la que conserva mayor cantidad de ejemplos, por regla general en buen estado de conservación. Relativamente poco conocido, a pesar de su calidad artística, es el que cubre el refectorio de la antigua Cartuja de Santa María de las Cuevas, actual Conjunto Monumental de La Cartuja, recientemente rehabilitado con ocasión de la Exposición Universal de Sevilla de 1992. Se conocía su existencia e importancia dentro de estas obras en madera, pero se desconocía su proceso de construcción y la autoría del mismo.

El hallazgo en el Archivo de Protocolos Notariales de Sevilla del contrato de dicha obra, nos sirve para precisar los autores y el año de su ejecución. Hasta el presente, se daba la de 1588 , fecha correspondiente a su definitiva instalación, que era recogida por el padre Rincón en su Protocolo de la Cartuja y que citaban todos

*. El presente trabajo parte del elaborado por el Equipo de Historiadores del Arte que participó en la realización del estudio histórico-artístico del Conjunto Monumental de La Cartuja, coordinado por Alfredo J. Morales y compuesto por José M. Baena, José R. Barros, $\mathbf{M}^{\mathbf{a}}$ Mercedes Fernández, Juan C. Hernández, Beatriz Maestre, Luis F. Martínez y Josefa Mata.

1. Los pioneros en el estudio de las armaduras fueron Gómez Moreno y Torres Balbás, sobre todo en cuanto a carpintería mudéjar. Más recientes son los estudios de Balbina Martínez Caviró y Basilio Pavón y los llevados a cabo por el mencionado Enrique Nuere. Este último ha dado un paso de gigante al intentar compaginar las aportaciones de los arquitectos y de los historiadores en el conocimiento y estudio de estos artesonados. Al respecto véanse, entre otros, NUERE, Enrique: La carpintería de lo blanco. Lectura dibujada del primer manuscrito de Diego López de Arenas. Madrid, 1985. Y La carpintería de armar española. Madrid, 1989. 
los autores que comentaban dicha techumbre ${ }^{2}$. No obstante, el artesonado fue contratado en 1587 por Diego Cerezo y Lucas de Cárdenas, ambos carpinteros de lo blanco en Sevilla ${ }^{3}$. El día 20 de febrero, fecha del contrato, los maestros se comprometían, a plena satisfacción de las partes, a terminar la obra en agosto del mismo año, obligándose, junto a tres oficiales y varios aprendices, a trabajar y no paralizar la obra hasta verla concluida desde el día que recibiesen el dinero y los materiales.

En cuanto a los artistas que intervienen en la obra son casi nulas las noticias referentes a otras actuaciones de los mismos. Sólamente se tienen referencias de la intervención en 1585 de Diego Cerezo en las obras de carpintería de la Casa de la Moneda. Poco más se sabe del carpintero Lucas de Cárdenas, quien sigue activo en la primera mitad del siglo XVII, trabajando en colaboración con artistas de la talla de Diego López de Arenas y del arquitecto Diego López Bueno ${ }^{4}$. Este hecho pudo facilitar la elección de estos maestros para la obra del monasterio cartujo ya que fueron muchos los carpinteros que pujaron para realizarla, primando en la elección su capacidad y experiencia, además del presupuesto presentado, que tan sólo era superior en 34 ducados sobre el más bajo y que rebajaba casi en trescientos la puja más alta ${ }^{5}$.

Las condiciones contractuales no responden exactamente al artesonado tal cual hoy se encuentra, pudiéndose deber éstas diferencias a intervenciones y reformas posteriores. No obstante, en líneas generales, tiene bastantes similitudes como más adelante se señalará.

$\mathrm{El}$ refectorio de La Cartuja ocupaba dentro del monasterio un lugar destacado, igual que ocurría en otras comunidades religiosas medievales. Estos estaban situados, por regla general, en una de las galerías del claustro, opuestas a la entrada del templo. Las dimensiones de los mismos eran considerables, dependiendo

2. V.P.D. José Martín Rincón. Protocolo del Monasterio de Nra. Sra. Santa María de las Cuevas. Tomo primero. Anales de los tres primeros siglos de su fundación ..., año 1400 en que se fundó y dotó ... Año 1744. Manuscrito inédito conservado en el Archivo de la Real Academia de la Historia de Madrid. Mss. (9/2098). Fol. 512.

3. Archivo Histórico Provincial de Sevilla. Leg. 2.385. Año 1587. Fol. 501 y ss.

4. El primero de ellos aparece indistintamente nombrado como Diego Cerezo o Diego Hernández Cerezo. GESTOSO Y PEREZ, José: Ensayo de un Diccionario de los artífices que florecieron en Sevilla desde el siglo XIII al XVIII inclusive. Tomo I. Sevilla, 1899. Pág. 53.

A Lucas de Cárdenas se le cita, junto a Francisco Fernández Navarrete, como autor de las obras de carpintería llevadas a cabo en unas casas de la collación de San Vicente en 1618. A.P.N.S. Escribanía de Pedro del Carpio. Oficio 4. 1618. Libro $3^{\circ}$, fol. 813-815. Véase además SANCHO CORBACHO, Heliodoro: "Contribución documental al estudio del arte sevillano". En Documentos para la Historia del Arte en Andalucía. Tomo II. Sevilla, 1928. Pág. 281. Y ARENILLAS, Juan Antonio: "Diego López Bueno, arquitecto del monasterio de Santa Paula de Sevilla (1615-1623)". En Archivo Español de Arte $\mathbf{N}^{\circ} 250$. Año 1990. Págs. 226 y 231

5. Otros maestros carpinteros que se citan en la escritura y que presentaron ofertas fueron Lope García, Juan Bautista, Alonso de Pineda, Melchor Gutiérrez y Hernán Arias. 
siempre del número de religiosos ${ }^{6}$. $\mathrm{Al}$ ser una estancia de gran importancia en la vida conventual siempre estuvo bien situada, citándosela constantemente como punto de referencia para la localización de otras dependencias del monasterio. Tenía acceso desde el claustro principal como ya se dijo y, en el caso de la Cartuja de Sevilla, también desde un pequeño claustrillo que articula las principales dependencias comunes del monasterio. Asimismo, comunicaba con las cocinas, desde donde se pasaba directamente la comida.

Esta dependencia se usaba sólamente para comer los días festivos o para celebrar algún acontecimiento importante, pues el resto del año la comunidad comía en solitario en sus respectivas celdas. El espacio interno se encontraba dividido por medio de un muro de poca altura con una puerta en el centro y dos ventanas laterales, que separaban la zona de monjes de la de legos, ocupando el prior una zona preferente.

El primitivo refectorio de La Cartuja se construyó en 1400, cuando fundó el convento don Gonzalo de Mena, aunque se agrandó dos veces debido al aumento que experimentó la comunidad ${ }^{7}$. En 1422 , se produjo la primera ampliación, a costa de un sector de la Capilla de la Magdalena, que había sido la primitiva iglesia de la comunidad. Dos años más tarde, en 1424, se contrataba la ejecución a destajo de la obra de carpintería de la techumbre del refectorio que, probablemente, sería una sencilla cubierta de vigas de madera o bien una techumbre de par-hilera, solución de precario equilibrio, pero fácil de construir. No obstante esta simplicidad, el refectorio contó desde un principio con un especial tratamiento ornamental, como demuestra la colocación en sus paredes de los escudos del fundador.

En torno a 1585 se produjo la segunda ampliación, siendo esta vez el claustro principal el que perdió espacio. Así, las arquerías de este último quedaron embutidas en la fachada sur del refectorio. Las obras debieron comenzar con anterioridad a mayo de 1585 , según se desprende del pleito que mantenía la casa de los Ribera, cofundadores del monasterio, con la comunidad por el patronazgo del mismo ${ }^{8}$. Con motivo de la ampliación se decoraron sus paredes con azulejos de superficie lisa con variados y coloristas motivos ornamentales. Idéntica decoración tenía el banco de obra, adosado a la pared, que recorría toda la estancia y que actualmente no se conserva. Asimismo, se han perdido las mesas de mármol blanco que, aún sin documentar, es tradición que procedían del antiguo convento Casa Grande de San Francisco de Sevilla, razón por la que estaba tallado el cordón franciscano en el frente .

6. Al respecto véase BRAUNFELS, Wolfgan: Arquitectura monacal en occidente. Barcelona, 1975.

7. CUARTERO Y HUERTA, Baltasar: Historia de la Cartuja de Santa María de las Cuevas, de Sevilla, y de su filial de Cazalla de la Sierra. Madrid, 1950. Tomo I. Pág. 133.

8. CUARTERO Y HUERTA, Baltasar: Historia de la Cartuja ... Op. cit. Tomo I. Pág. 493.

9. CUARTERO Y HUERTA, Baltasar: Historia de la Cartuja ... Op. cit. Tomo I. Pág. 584. 
Las paredes estaban decoradas, a su vez, con grandes lienzos que en el testero, lugar reservado al prior, se complementaban con una rica decoración de pintura mural. Del primitivo refectorio se conserva la portada que lo comunica con el citado claustrillo y el púlpito, abierto como aquella en el muro oriental y ornamentado con yeserías de tracería gótica. Dicho elemento es común a todas las comunidades religiosas y, más en concreto, a la cartujana, pues la lectura, tanto individual como colectiva, es base fundamental en la vida espiritual de los monjes.

En el siglo XIX, cuando el edificio se acondicionó para fábrica de loza, el refectorio se utilizó en un primer momento como taller de fabricación, dividiéndose más tarde en dos pisos para destinarlo a almacén de la loza ya concluida. Debido a esta nueva reutilización del edificio desapareció el banco corrido que rodeaba la estancia, así como el tabique de separación entre la zona de legos y la de monjes. También se abrieron nuevos vanos en las paredes, perdiéndose con ello parte de los zócalos de azulejos que decoraban los muros de la sala.

Por fortuna se conservó el magnífico artesonado que la cubre, como conclusión al proceso de renovación de dicha estancia. En la bibliografía tradicional se mantiene que es de madera de alerce, árbol de la familia de las coníferas. Estos árboles tienen la particularidad de que son las únicas coníferas europeas de hoja caduca, con una madera dura muy empleada en carpintería aplicada a la construcción. El Padre Rincón, al referirse al artesonado en su Protocolo, afirma que esta madera era incorruptible y que resultaba imposible que apareciesen polillas y otros animales que perjudicasen a la madera ${ }^{10}$. No obstante, el análisis científico de dicha madera ha demostrado que, en realidad, se trata de pino, concretamente de la especie "Pinus Sylvestris", conocido comercial y vulgarmente como Pino Norte o Flandes, presentando los anillos de crecimiento una disposición muy regular y homogénea ".

El artesonado responde estructuralmente a las armaduras de par y nudillo. Esta solución es una de las más características y la más sencilla de las cubiertas de madera a dos aguas. Los pares se colocan distanciados unos de otros y su hueco lo viene a cubrir una tablazón llamada tabica. Para evitar que los pares se cimbreen se colocan horizontalmente los nudillos que, enlazados unos con otros por medio de la tablazón, dará lugar al almizate o harneruelo, parte horizontal de la techumbre que junto con la alfarda, la parte que cuelga en los laterales, da a la armadura su característica forma de artesa ${ }^{12}$.

10. El padre Rincón al referirse al artesonado comenta que es "...Costoso por su fabrica y no menos por lo incorruptible y precioso de la Madera de Alarce a que el criador dio perpetuo privilegio para no admitir nidos de arañas, polillas ni otras semejantes sabandijas sus enemigas ...". MARTÍN RINCÓN, José: Protocolo del Monasterio ... Op. cit. Fol. 512.

11. El análisis de la madera fue realizado en 1989 por el Laboratorio de Biología del Instituto de Conservación y Restauración de Bienes Culturales del Ministerio de Cultura.

12. Otros autores mantienen que la palabra artesonado proviene del francés "artison", que significa carcoma, de donde deriva artesón o agujero, siendo válida esta definición sólamente cuando se refiere a armaduras decoradas con artesones o casetones, muy utilizadas en el Renacimiento. 
La armadura del refectorio es de las llamadas de lima, una variedad de las de par y nudillo, aunque su flanco meridional carece de faldón, condición que se expresa en el documento de contrato, como se señalará más adelante. Aquellas se caracterizan por tener cuatro paños o faldones en vez de los dos, que ofrecen las de par y nudillo, en correspondencia con los lados mayores de la habitación. En el caso del artesonado que nos ocupa cuenta con una sóla lima. El almizate y alfarda se decoran con labor de lacería, formando bandas entrelazadas, siempre rectas o en ángulo que van formando polígonos estrellados.

Pasando a analizar el artesonado de la cartuja sevillana, puede comprobarse que Diego Cerezo y Lucas de Cárdenas, se ajustaron prácticamente a todas las condiciones estipuladas en el contrato, sobre todo en lo referente a su estructura. Antes de comenzar la obra los carpinteros se comprometieron a desmontar la armadura antigua, especificándose que se hiciera por tramos, debiéndose cuidar este despiece para poder aprovechar la madera y clavos para otras obras y, probablemente también, en los andamios e incluso en los nudillos de la nueva pieza. El ritmo de estos trabajos debía acoplarse al de la factura del nuevo artesonado para no dejar totalmente al descubierto el refectorio. Los andamios debían ir situados por encima de la Capilla de Santa Ana, lugar por el que tenía que colocarse la nueva armadura, trabajando los operarios en el patio y soportales existentes junto a la puerta de la iglesia.

Según la obligación del contrato, una vez desmontada la antigua armadura se comenzaron a labrar los nudillos o maderos en forma de cubo que irían empotrados en la pared, separados entre sí una vara, -casi un metro-, colocándose dos en los ángulos para salvar la estructura rectangular. Sobre ellos se situaría la solera que debía tener "una sexma de grueso por una tercia de ancho", que equivaldría aproximadamente a $0,39 \times 0,278 \mathrm{~m}$., ensamblada por el sistema de cuadrado a media madera y clavada a los canecillos, ensamble de similares características al inglete. A continuación se debían labrar los canes decorados sus frentes con temas de cartones, rétulos y florones que medirían una sexma de grueso y palmo y medio de alto $-0,39$ x 0,312 m.-. El siguiente paso consistía en desbastar y ubicar el arrocabe entre los canes.

A partir de aquí se empezarían a labrar los tirantes, sin establecer el número necesario, dejándolo a juicio del maestro carpintero. Aquellos irían a su vez decorados con temas de lazo apeinazado para, posteriormente, colocarse sobre los canecillos. El paso siguiente era labrar los testeros, estableciendo uno de limas con sus péndolas y, el del muro sur a mojineje, es decir sin faldón o gualdera, característica de las cubiertas de lima, ya que ocultaría parte de la ventana que ilumina el recinto, utilizando en sustitución de éste una especie de zapata, quedando cortado a la altura de la ventana ${ }^{13}$.

13. " ... y digo que en el testero que a de ser amoxinete que esta la vedriera meta unos nudillos apretados con su yeso y por la orden u acuesto del arrocabe para que ate de una banda y otro el arrocabe con la vedriera serrando este arco cabe por los frentes de la vedriera por la propia orden y luego labre unos estribos labrados ...". A.H.P.S. Año 1587. Leg. 2.385. Fol. 504. 
Una vez realizado todo este trabajo a pie de obra se montaría la armadura propiamente dicha, procediéndose a su decoración. Esta, a base de lazo, se debía clavar sobre una tabica realizada en madera tosca, tabicándose también hacia el exterior para que el maestro albañil pudiera colocar las tejas. El tipo de lazo empleado es el de lefe, rueda de lazo de diez, compuesta en su totalidad con azafates redondos ${ }^{14}$. Además de la decoración de lazo, el almizate debía ir decorado con cinco piñas de mocárabes de "siete y cinco" sujetas a este por medio de un vástago metálico.

Respecto a las especificaciones del contrato el artesonado actual ofrece coincidencias evidentes, caso de la ornamentación y de la falta del arrocabe en el testero del muro sur, solución que da a la pieza aspecto de inacabada ${ }^{15}$. En cambio, no coincide en la utilización de tirantes de madera, sustituidos en este caso por otros de hierro embutidos en el arrocabe y apoyando directamente sobre el muro. La utilización de tirantes de hierro es poco frecuente en las cubiertas de la zona sevillana, siendo más usual los de madera, decorados también con temas de lazo, tal como se recoge en el pliego de condiciones, y que alcanzarían un gran desarrollo en Andalucía occidental. El empleo de tirantes, ya fueran de un tipo o de otro, era imprescindible en el caso de grandes cubriciones, a pesar de ser los estribos los que recogen los empujes. Este es el caso del correspondiente al refectorio de Santa María de las Cuevas, donde el espacio a cubrir es, aproximadamente, de siete metros de ancho por treinta de largo, siendo necesaria la utilización de seis tirantes que compartimentan la cubierta. Al no colocarse los tirantes de madera tampoco presenta los canes que serían precisos para apoyarlos.

En cuanto a la decoración, sigue los esquemas mudéjares que tanto arraigo tuvieron en la zona, si bien combinados con elementos manieristas. El primer estilo decorativo se aprecia en el almizate y alfarda, donde las estrellas de diez puntas se repiten por todo el artesonado, con azafates redondos que forman candilejos irregulares de cinco puntas, terminando en medios círculos o semirruedas formadas por los peinazos en la base de la alfarda. Las tres piñas de mocárabes que se conservan responden a este mismo tipo decorativo, si bien no responden en número a las descritas en el documento. Aquellas alternan con dieciseis piñas, de las diecisiete que debieron existir, de menor tamaño y de traza manierista, policromadas y doradas que contrastan fuertemente con los temas mudéjares. Idéntica decoración presentan los escudos del fundador don Gonzalo de Mena, dos de ellos en el almizate y catorce alrededor de la alfarda y situados en las medias ruedas

14. Los azafates son cada una de las piezas alargadas que forman la rueda del lazo cuando su cabeza forma un ángulo obtuso. Se diferencian de los azafates harpados en que estos tienen la cabeza recta o con punta en el centro.

15. "... y digo que el testero que a de ser a moxinete le eche dos medios sercos que alleguen a la vedriera y les eche dos o tres aspas a cada uno para que sirvan de tirante junto a la vedriera porque quitara la vista y si se a de echar una tirante de hierro la eche ...". A.H.P.S. Año 1587. Leg. 2.385. Fol. 504. 
de lazo ${ }^{16}$. Probablemente estos elementos decorativos, junto con las rosetas de talla inscritas en un cuadrado de la alfarda, se deban a una incorporación posterior al proyecto, tal vez, suscitada durante el proceso de construcción.

Varios artesonados se conservan en Sevilla y su provincia fechados en los años finales del siglo XVI y principios de la centuria siguiente, años en los que estaba activo el carpintero Diego López de Arenas. En su tratado Breve compendio de carpintería de lo blanco y tratado de alarifes, obra manuscrita fechada en 1619 y publicada en Sevilla unos años más tarde, reproduce un artesonado de lazo de diez, similar al de la Cartuja ${ }^{17}$. De la misma decoración de lazo de diez es el existente en el convento de Madre de Dios, si bien este es de cinco paños y ochavado. Fue contratado en 1564 por los maestros Francisco Ramírez, Alonso Ruiz y Alonso del Castillo ${ }^{18}$. No obstante, estilísticamente al que más se parece en su decoración es el de la nave principal del convento de San Pablo y Santo Domingo de Ecija, fechable en los años finales del siglo XVI, ya que hacia 1595 el edificio estaba a falta de cubrirse ${ }^{19}$.

Por regla general las armaduras antiguas conservadas, y en concreto la del refectorio de la Cartuja sevillana, se encuentran en buen estado de conservación a pesar de las pudriciones, el ataque de los insectos xilófagos, debidos principalmente a la humedad, y a las pérdidas de algunos elementos. Esta buena conservación se debe sin duda al buen tratamiento de la madera empleada, con un proceso de secado natural, y a la calidad de la misma que presupone la existencia de grandes carpinteros conocedores de su oficio, artistas que sabiamente supieron fundir la decoración tradicional con las nuevas corrientes estilísticas, como lo demuestran en el artesonado del refectorio cartujo.

16. El escudo presenta un campo de azur con cinco estrellas de oro, puestas en sotuer, con bordura de gules con ocho bezantes de plata, cargado cada uno con tres fajas de azur, por su apellido Roelas. Se remata con capelo alusivo a su cargo y una cruz.

17. Edición facsímil con nota previa de Enrique Nuere. Valencia, 1982. Asimismo, véase TOAJAS ROGER, $\mathrm{M}^{\mathbf{a}}$ Angeles: Diego López de Arenas, Carpintero, alarife y tratadista en la Sevilla del siglo XVII. Sevilla, 1989.

18. VALDIVIESO GONZALEZ, Enrique y MORALES MARTINEZ, Alfredo J.: Sevilla oculta. Monasterios y conventos de clausura. Sevilla, 1980. Pág. 105.

19. HERNANDEZ DIAZ, José; SANCHO CORBACHO, Antonio y COLLANTES DE TERAN, Francisco: Catálogo Arqueológico y Artístico de la provincia de Sevilla. Tomo III. Sevilla, 1951. Pág. 160. 


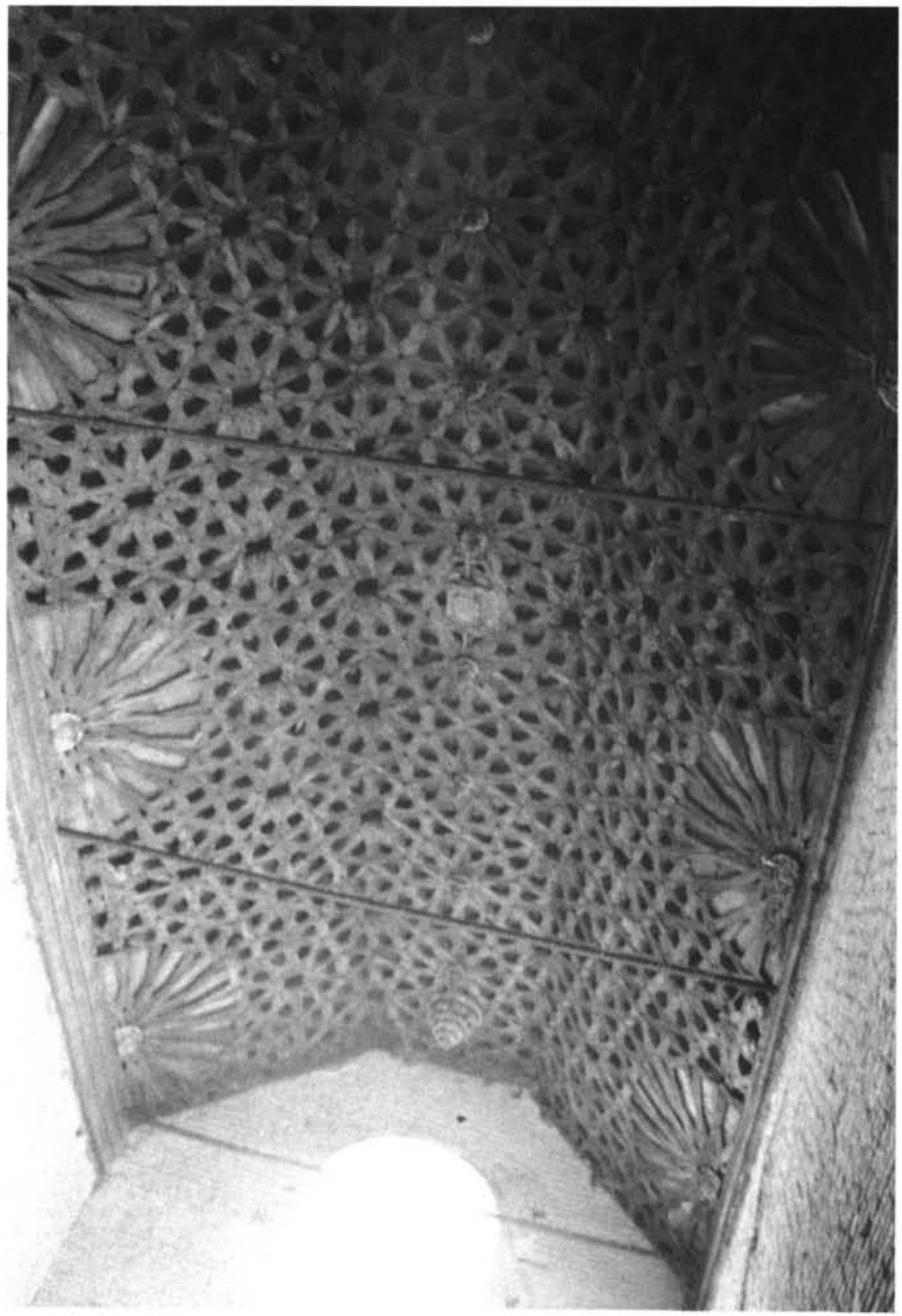

Diego Cerezo y Lucas de Cárdenas. Artesonado del refectorio de La Cartuja de Santa María de las Cuevas. 


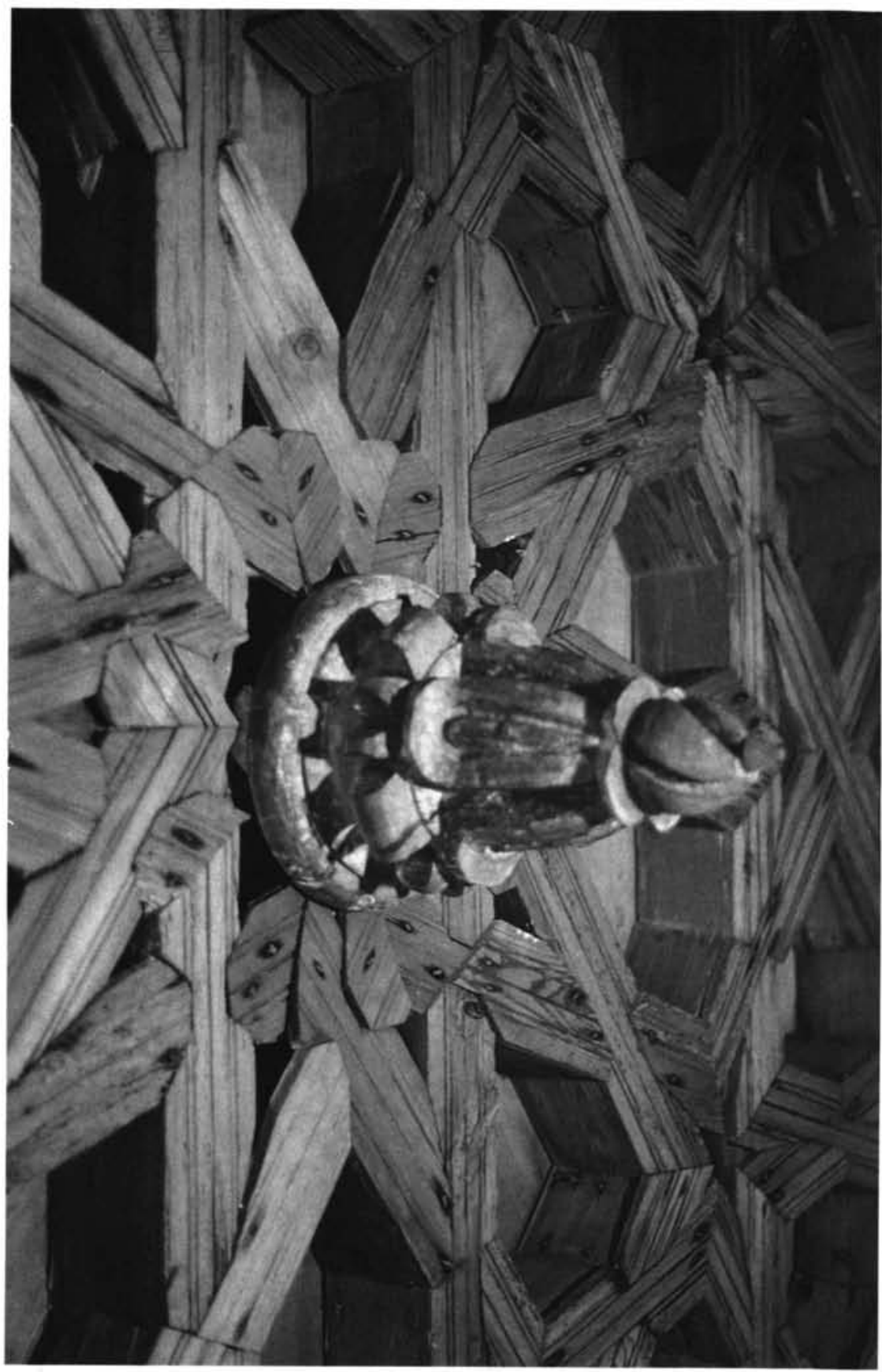

